\title{
A decade of science and engineering of composite materials at the North West Composites Centre, University of Manchester, UK
}

\author{
Constantinos Soutis ${ }^{1}$ (D)
}

Received: 31 October 2016/Accepted: 2 November 2016 / Published online: 1 December 2016

C) The Author(s) 2016. This article is published with open access at Springerlink.com

\begin{abstract}
The University of Manchester, School of Materials has a large multidisciplinary research programme on polymers, composites and carbon-based materials. This takes place through fundamental studies of structure-property relationships for these materials, including controlled synthesis and processing, and effects of structure andnano-, meso- and macro-scale morphology on physical properties and engineering applications.
\end{abstract}

Keywords Composites · Graphene - Textiles · Damage tolerance $\cdot$ Non-destructive techniques · SHM

High performance polymer composites have gained great acceptance in areas where their lightweight and exceptional strength outweigh cost issues, for example in the aerospace sector. Now, with developments in materials, automation and low-cost processing technologies, composites can be used economically in a growing number of applications in automotive, wind/tidal energy, marine, rail, civil-infrastructure, medical and recreational industries.

The University of Manchester, School of Materials has a large multidisciplinary research programme on polymers, composites and carbon-based materials. This takes place through fundamental studies of structure-property relationships for these materials, including controlled synthesis and processing, and effects of structure and nano-, meso- and macro-scale morphology on physical properties and engineering applications. Established in February 2006, the Northwest Composites Centre (NWCC) received a £2.1 million grant from the Northwest Science Fund, administered by the Northwest Regional Development Agency (NWDA). Further investment of over $£ 8$ million from the NWDA and the University of Manchester has resulted in the creation of the National Composites Certification and Evaluation Facility

Constantinos Soutis

constantinos.soutis@manchester.ac.uk

1 Aerospace Research Institute, University of Manchester, Manchester M13 9PL, UK 
(NCCEF), an UKAS (ISO-17025) accredited laboratory for the testing and evaluation of composite materials.

NWCC partners with the National Composites Centre (NCC) and the Composites UK and participates in major research programmes including the EPSRC Centre for Innovative Manufacturing in Composites (cimcomp.ac.uk), EPSRC SuperGen, EPSRC-JLR, AMSCII Composites Innovation Cluster and the Aerospace Technology Institute (ATI). Leading research areas in the Centre include multi-scale modelling \& imaging, novel testing, NDT, Structural Health Monitoring, novel multi-scale textile architectures for affordable damage tolerant composites, robotic fibre placement and 3D textile preforming and nanomaterial enhanced composites. The Centre currently hosts over $50 \mathrm{PhD}$ students and Post-doctoral researchers with funding provided by the Research Councils UK, Government and industry.

This ACMa issue is a refereed compilation of selected papers by authors who are all workers of the Centre, presenting elements of their current research work, proposing graphene as a way of introducing functionality in advanced composites, 3D fibre architectures to improve damage resistance and tolerance of such systems that in order to evaluate their structural integrity, non-destructive techniques (NDT) and real time structural health monitoring (SHM) will be required. There are three papers focusing on nano-composites that highlight the challenges faced in mixing and dispersing graphene, its impact on mechanical, thermal and electrical properties and how analytical and numerical modelling could be used to identify the optimal shape, size and amount of the nano-filler. The reader will observe some good progress is made on analysis and experiment that could lead to improved design with several questions still unanswered requiring further effort and investment. Nine articles on $2 \mathrm{D}$ and $3 \mathrm{D}$ woven composites deal with manufacturing challenges, propose ways of simulating the mechanical behaviour and demonstrating the effect of fibre architecture on performance of flat woven laminates, T-joints reinforced with veils and through-the-thickness stitching and cylindrical tubes. Fabrication induced defects and in-service damage degrades the mechanical properties and reduces life, so early non-destructive detection is essential in preventing premature catastrophic failures; four papers are dedicated to NDT and real time structural health monitoring concepts that could be implemented in composite engineering applications, avoiding sudden catastrophic failures, which can be costly.

The authors of each paper begin with a comprehensive literature review that describes progress made on the relevant topic, identifying remaining challenges and then delving into the natural characteristics and behaviour of a particular composite material system, probing and examining such concepts and relationships as structure and design from the very small to the very large. What emerge are the evolution of a number of neighbouring disciplines in processing, manufacturing and mechanical design; experimentation and analysis; mathematical and continuum modelling; constitutive and physical modelling (micro-mechanics or damage mechanics); computational mechanics and virtual simulation aided by careful experimental observations. It is envisioned that new strategies for designing damage resistant and tolerant composite materials and structures may become available, if we first develop and then synergistically combine new capabilities enabling in-service damage detection and characterisation, health monitoring and structural prognosis. The rapid development of numerical codes and experimental techniques make possible not only the robust modelling behind the design of advanced composites with improved behaviour in critical operational conditions, but also for establishing sound, reliable SHM methods and strategies. The challenges will be an integration of modelling the design of composite structures, as well as process modelling, together with SHM and repair strategies. Moreover, SHM may also 
promote a "self-healing" reaction in case of a particular damage, keeping the structural integrity, at least for a given period of time. The assurance of structural reliability of aircraft systems will greatly enhance confidence in their safety, reduce the probability of premature failures, and diminish the costs of operation and maintenance.

We hope you will enjoy reading these papers and we would like to take this opportunity to thank the authors who submitted them and of course the referees who helped ensure that the journal's high standards were maintained. Last but not least we would like to acknowledge our gratitude to Editor-In-Chief, Dr Peter Beaumont for his kind invitation to contribute this Special Issue and his support throughout the reviewing process ensuring its smooth compilation and publication.

\section{Guest Editors}

\section{Professor Costas Soutis FREng \\ Professor Prasad Potluri}

Manchester, 31 October 2016

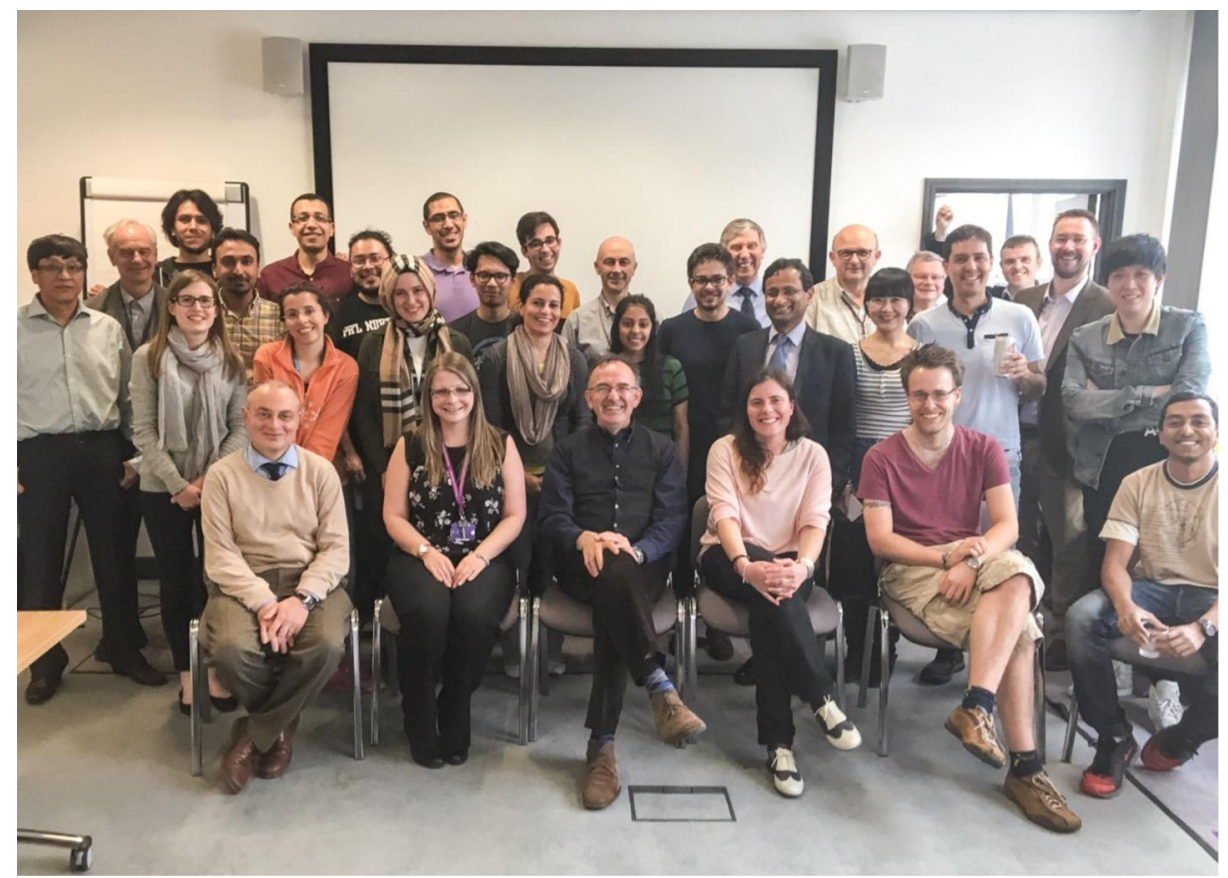

University of Manchester Composites group

Open Access This article is distributed under the terms of the Creative Commons Attribution 4.0 International License (http://creativecommons.org/licenses/by/4.0/), which permits unrestricted use, distribution, and reproduction in any medium, provided you give appropriate credit to the original author(s) and the source, provide a link to the Creative Commons license, and indicate if changes were made. 